

\section{DISCLAIMER}

This report was prepared as an account of work sponsored by an agency of the United States Government. Neither the United States Government nor any agency Thereof, nor any of their employees, makes any warranty, express or implied, or assumes any legal liability or responsibility for the accuracy, completeness, or usefulness of any information, apparatus, product, or process disclosed, or represents that its use would not infringe privately owned rights. Reference herein to any specific commercial product, process, or service by trade name, trademark, manufacturer, or otherwise does not necessarily constitute or imply its endorsement, recommendation, or favoring by the United States Government or any agency thereof. The views and opinions of authors expressed herein do not necessarily state or reflect those of the United States Government or any agency thereof. 


\section{DISCLAIMER}

Portions of this document may be illegible in electronic image products. Images are produced from the best available original document. 


\title{
IMPROVED FIRST ORDER ANALYSIS OF THE PLASTIC STABILITY OF DIFFUSE PATCHES IN TUBES AND SHEETS \\ (LWBR Development Program)
}

E. Duncombe

August 1973

Contract AT-11-1-GEN-14

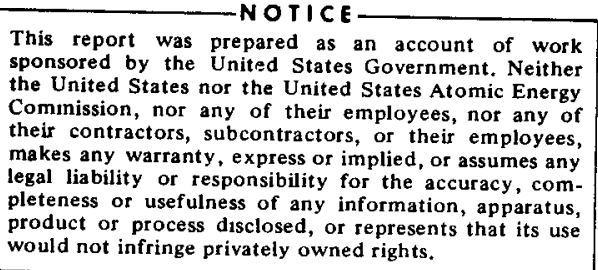

would not infringe privately owned rights.

\author{
Printed in the United States of America \\ Available from the \\ National Technical Information Service \\ U. S. Department of Commerce \\ 5285 Port Royal Road \\ Springfield, Virginia 22151
}

\section{BETTIS ATOMIC POWER LABORATORY, PITTSBURGH, PA., OPERATED FOR THE U. S. ATOMIC ENERGY COMMISSION BY WESTINGHOUSE ELECTRIC CORPORATION}




\section{NOTICE'}

This report was prepared as an account of work sponsored by the United States Government. Neither the United States, nor the United States Atomic Energy Commission, nor any of their employees, nor any of their contractors, subcontractors, or their employees, makes any warranty, express or implied, or assumes any legal liability or responsibility for the accuracy, completeness or usefulness of any information, apparatus, product or process disclosed, or represents that its use would not infringe privately owned rights. 


\section{CONTENTS}

Page

I. INTRODUCTION

II. MATERIAL PROPERTIES

III. ANALYSIS METHODS

A. Definition of Stability

B. The Variable Being Examined

C. Boundary Conditions

2

IV. CHARACTERISTIC EQUATION FOR STABILITY 3

V. DISCUSSION OF RESULTS

A. Roots of the Characteristic Equation 4

B. Critical Values of $\gamma$ and $\mathrm{m} \quad 5$

VI. CONCLUSIONS

REFERENCES

APPENDIX - Derivation of the Characteristic
Equation for Stability

\section{LIST OF ILLUSTRATIONS}

$\underline{\text { Figure }}$

Title

1 Locally Thinned Region of a Tube Subjected to Internal Gas Pressure and End Loads

2 Roots of Equation for Neck Growth, $\mathrm{R}=\mathrm{P}=1, \mathrm{~m}=10^{-\mathrm{b}}$

3 Roots of Equation for Neck Growth, $R=P=1, m=0.01$

4 Roots of Equation for Neck Growth, $R=P=1, m=0.25$

5 Roots of Equation for Neck Growth, $\mathrm{R}=\mathrm{P}=1, \mathrm{~m}=1$

6 Roots of Equation for Neck Growth, $\mathrm{R}=2, \mathrm{P}=4, \mathrm{~m}=10^{-5}$

7 Roots of Equation for Neck Growth, $R=4, P=4, m=10^{-5}$

8 Absolute and Effective Stability Criteria, $\mathrm{R}=\mathrm{P}=\mathrm{l}$

9 Absolute and Effective Stability Criteria, $R=2, P=4$

10 Absolute and Effective Stability Criteria, $R=P=4$ 
Previously published work on plastic instability of patches in tubes and sheets under biaxial stress conditions is extended by the use of improved first order approximations and more general material property relationships The former relates to the inclusion of first order changes in the strain direction inside the patch and the latter relates to the inclusion of material anisotropy and strain rate sensitivity (defined in the text) Inclusion of strain directors changes the form of the characteristic equation for stability from first order to second order but both roots are real for all practical cases For some stress conditions the largest root is positive at all values of strain hardening parameter (defined in the text) However, the values of strain hardening at which the largest root becomes significantly positive are very close to those reached for zero root when changes in strain directors are ıgnored By this significance test the stability also increases rapidly above a strain rate sensitivity of about 01

\section{IMPROVED FIRST ORDER ANALYSIS OF THE PLASTIC STABILITY OF DIFFUSE PATCHES IN TUBES AND SHEETS (LWBR Development Report)}

\section{E. Duncombe}

\section{INTRODUCTION}

Plastic instability in tubes and sheets may be defined as a condition whereby a small localized region, which is thinner than the surrounding material of nominal size, tends to decrease progressively in thickness relative to its surroundings. This condition is similar to the corresponding necking instability of bars in a uniaxial stress condition However, in this case the stress field must be taken into account, for example, the two principal membrane stresses acting in the plane of the sheet. The presence of significant stresses in the thickness direction also influences stabrlity, but this situation will not be treated here. Likewise, bending moments and shear stresses in the sheet are assumed small enough to have only minor effects. The stability against progressive opening of grooves has been examined in a previous paper (Reference 1). Also, analysis of conditions under which localized thinned patches can form in tubes and sheets under biaxial stress conditions has been carried out by a number of previous workers (References 2, 3, and 4). The stability conditions covered here relate to the latter mode with certain additional effects. The added effects are (1) the influence of strain rate sensitivity, (2) anisotropic material properties, and (3) the fact that strain rate directors themselves vary with the altered stress condition inside the patch.

\section{MATERIAL PROPERTIES}

In addition to the biaxial stress condition the other major factors entering into stability are the material constitutive properties. These can be expressed in terms of differentials as follows:

$$
\frac{d \sigma}{\sigma_{g}}=m \frac{d \dot{\varepsilon}_{g}}{\dot{\varepsilon}_{g}}+n \frac{d \varepsilon_{g}}{\varepsilon_{g}}
$$

where $\sigma_{g}$ is the generalized stress, a function of the principal stresses, and $\epsilon_{g}$ is the generalized strain in the material, being a function of the three principal strains. These will be defined later. $\AA$ dot over a variable indicates the time rate of change. Symbols $m$ and $n$ for present purposes can be considered as numbers which are characteristic of the material in the neighborhood of $\sigma_{g}, \dot{\epsilon}_{g}, \epsilon_{g}$ but not necessarily outside this neighborhood. The parameter $m$ is usually called the strain rate sensitivity and the parameter $n$ the strain hardening exponent. It is often convenient to replace $n / \epsilon_{g}$ in Equation (1) by a single parameter $\gamma$, called the strain hardening parameter. The generalized stress $\sigma_{g}$ in the constitutive relation in Equation (1) is defined in a manner similar to Hill's definition (Reference 5) as

$$
\sigma_{8}=\left[\frac{R\left(\sigma_{h}-\sigma_{t}\right)^{2}+R P\left(\sigma_{t}-\sigma_{\ell}\right)^{2}+P\left(\sigma_{\ell}-\sigma_{h}\right)^{2}}{R P+P}\right]^{1 / 2}
$$

where $\sigma_{h}, \sigma_{t}$ and $\sigma_{\ell}$ are the principal stresses in thickness, width, and length directions and the anisotropic parameters $R$ and $P$ determine the material strength in each direction. The generalized strain rate $\dot{\epsilon}_{g}$ is defined by the work law:

Rate of plastic work $=\sigma_{g} \dot{\varepsilon}_{g}=\sigma_{h} \dot{\varepsilon}_{h}+\sigma_{t} \dot{\varepsilon}_{t}+\sigma_{\ell} \dot{\varepsilon}_{\ell}$ 
Assuming orthogonality of strain rate components to the locus of constant $\sigma_{g}$ in stress space (Reference 6), it can be shown that

$$
\begin{aligned}
& \dot{\varepsilon}_{h}=\left(\frac{\partial \sigma_{g}}{\partial \sigma_{h}}\right) \dot{\varepsilon}_{g}=D_{h} \dot{\varepsilon}_{g} \\
& \dot{\varepsilon}_{t}=\left(\frac{\partial \sigma_{g}}{\partial \sigma_{t}}\right) \dot{\varepsilon}_{g}=D_{t} \dot{\varepsilon}_{g} \\
& \dot{\varepsilon}_{\ell}=\left(\frac{\partial \sigma_{g}}{\partial \sigma_{l}}\right) \dot{\varepsilon}_{g}=D_{\ell} \dot{\varepsilon}_{g}
\end{aligned}
$$

which defines the directors $D_{h}, D_{t}$, and $D_{\ell}$ If $\sigma_{h}$ is small enough to be neglected, the expression for the directors $D_{h}, D_{t}$, and $D_{\ell}$, obtained by differentiating Equation(2), is as follows

$$
\begin{aligned}
& D_{h}=\left[\frac{-(R+P \alpha)}{R P+P}\right] \beta \\
& D_{t}=\left[\frac{-R P(1-\alpha)+P \alpha}{R P+P}\right] \beta \\
& D_{\ell}=\left[\frac{-R P(1-\alpha)+P \alpha}{R P+P}\right] \beta
\end{aligned}
$$

where

$$
\begin{aligned}
\alpha & =\sigma_{l} / \sigma_{t} \\
\beta & =\sigma_{t} / \sigma_{g} \\
& =\left[(R P+P)^{1 / 2} /\left(R+R P(\alpha-1)^{2}+P \alpha^{2}\right)^{1 / 2}\right]\left(\frac{\sigma}{|t|}\right)
\end{aligned}
$$

It is assumed here that $\dot{\epsilon}_{\mathrm{h},} \dot{\epsilon}_{l}$ and $\dot{\epsilon}_{\mathrm{t}}$ are the total strain rates, $1 e$, the additional elastic contribution to the strain rates in Equations (4), (5), and (6) are assumed to be negligible compared with plastic strains

At this stage it is convenient to define for later use the second derivatives of $\sigma_{g}$, as follows

$$
\begin{aligned}
& D_{l \ell}=\left(\frac{\partial^{2} \sigma_{g}}{\partial \sigma_{\ell}^{2}}\right) \sigma_{g}=1-D_{l}^{2} \\
& D_{t t}=\left(\frac{\partial^{2} \sigma_{g}}{\partial \sigma_{t}^{2}}\right) \sigma_{g}=\frac{R P+R}{R P+P}-D_{t}^{2}
\end{aligned}
$$

$$
D_{\ell t}=D_{t \ell}=\left(\frac{\partial^{2} \sigma_{g}}{\partial \sigma_{\ell} \partial \sigma_{t}}\right) \sigma_{g}=\frac{-R P}{R P+P}-D_{\ell} D_{t}
$$

It should be noted that the constitutive relation in Equation (1), in the absence of further information, should in fact be written as a vector relationship between the principal stresses $\sigma_{\mathrm{h}}$, $\sigma_{\mathrm{t}}$ and $\sigma_{\ell}$ and the principal strain rates $\dot{\boldsymbol{\epsilon}}_{\mathrm{h}}, \dot{\boldsymbol{\epsilon}}_{\mathrm{l}}$ and $\dot{\epsilon}_{\ell}$ The alternative procedure of using a single equation via a stress function $\sigma_{\mathrm{g}}$ defined by Equation (2) may be regarded as merely a two-parameter ( $1 \mathrm{e}, \mathrm{R}$ and $\mathrm{P}$ ) fitting process which is not exact, but which is usually sufficiently accurate (Reference 6)

\section{ANALYSIS METHODS}

Necessary prerequisities to the analysis of stability are (1) an exact definition of stability, (2) a selection of the variable being examined for stability, and (3) definition of the boundary conditions relating stress and strain conditions outside the patch and those inside These will now be considered

\section{A. Definition of Stability}

The criterion of Lyapunov, usually described as his "first method" (Reference 7), will be used here Briefly, the equilibrium points of the system are furst determined and then the dynamic equations are linearized into homogeneous equations for small departures from these equilibrium points The eigenvalues (latent roots) of these lineanzed equations are then determined If any of the eigenvalues are positive the system is unstable The equations themselves are strictly applicable only to local regions around the equihbrium point over which the linearization is valid, and thus this definition is sometımes referred to as "stability in the small" rather than stability "in the large" which would cover the global situation

\section{B. The Variable Being Examined}

The specific variable chosen here is the difference $\delta \epsilon_{\mathrm{g}}$ between generalized strain in the necked region and that in the surrounding unnecked region (Figure 1) The symbol $\delta$ is always used hereafter to denote a difference between the value of some parameter inside the necked region and the value in the material outside this region at the same instant of time Variables other than $\delta \epsilon_{\mathfrak{g}}$ could be chosen For instance, it would be feasible to choose the difference in thickness $8 \mathrm{~h}$ Alternatively, the dif- 
ference in thickness strain could be chosen, i.e., $\delta \epsilon_{\mathrm{h}}$. The three variables are related as follows:

$$
\begin{gathered}
\delta \dot{\varepsilon}_{h}=\delta\left(D_{h} \dot{\varepsilon}_{g}\right)=D_{h} \delta \dot{\varepsilon}_{g}+\sum_{i} \dot{\varepsilon}_{v s}\left(\frac{\partial D_{h}}{\partial \sigma_{i}}\right) \delta \sigma_{1} \\
\delta \dot{\varepsilon}_{h}=\delta\left(\frac{\dot{h}}{h}\right)=\left(\frac{1}{h}\right) \delta \dot{h}-\left(\frac{\dot{h}}{h}\right) \delta h=\frac{1}{h} \delta \dot{h}-\dot{\varepsilon}_{h} \delta \varepsilon_{h}
\end{gathered}
$$

Conditions for the stability of these variables would be expected to be similar. They are not identical, since the new variables involve integrals of the variable $\delta \dot{\epsilon}_{g}$, which are formally infinite when the latent roots determining $\delta \dot{\epsilon}_{g}$ are zero. For instance, if

$$
\delta \dot{\varepsilon}_{g}=K \exp [\lambda t]
$$

then $\lambda=0$ when

$$
\left[\begin{array}{ll}
\frac{d}{d t} & \delta \dot{\varepsilon}_{g}
\end{array}\right]_{t=0}=0
$$

However, $\lambda<0$ when

$$
\frac{d}{d t}\left[\delta \dot{\varepsilon}_{g}+\int \delta \dot{\varepsilon}_{g} d t\right]_{t=0}=0
$$

\section{Boundary Conditions}

The boundary conditions assumed here are illustrated in Figure 1 which shows a tube under internal pressure and end loads. The basic assumptions are that the principal stresses outside the thinned region are amplified inside the region by the ratio of thickness in these locations.

The possibility of localized thinning is most evident in the case of drawing processes (References 8 and 9) in which case the locally thinned patches would be expected to become more highly stressed as they become thinner. In the case of flat plates localized thinning would lead to unloading, and in such cases other instability mechanisms such as the growth of groove-like defects may be expected to dominate (Reference 1). Internally pressurized tubes could develop instabilities in either mode depending on dimensions and material properties. Usually in pressurized tube tests the initial instability is diffuse (patch-type) although the final tear may be associated with groove instability.
Adopting the boundary assumptions as stated, the following relationships apply to the membrane stresses $\sigma_{l}$ and $\sigma_{t}$ :

$$
\begin{aligned}
& \frac{\delta \sigma_{\ell}}{\sigma_{\ell}}=-\frac{\delta A_{\ell}}{A_{\ell}}=\delta \varepsilon_{\ell} \\
& \frac{\delta \sigma_{t}}{\sigma_{t}}=-\frac{\delta A_{t}}{A_{t}}=\delta \varepsilon_{t}
\end{aligned}
$$

where $\delta A_{1}$ is the difference in area of cross section over which the applied force in direction $i$ acts. The second equality in each of Equations (18) and (19) arises from the assumption of constant volume.

\section{CHARACTERISTIC EQUATION FOR STABILITY}

It is shown in the Appendix that the constitutive Equation (1) and the boundary conditions, Equations (18) and (19), together with the expression for generalized stress, Equation (2), and the strain rate Equations (5) and (6) are sufficient to specify a set of homogeneous equations in the variables $\delta \sigma_{g}$, $\delta \sigma_{\ell}, \delta \sigma_{\mathfrak{t}}, \delta \epsilon_{\mathrm{g}}, \delta \epsilon_{\ell}, \delta \epsilon_{\mathrm{t}}$. This leads to a characteristic equation in $\epsilon_{g}$ which is of the form

$$
\left(A S^{2}+B S+C\right) \delta \tilde{\varepsilon}_{g}=0
$$

where $S$ stands for the derivative $d / d t$. The coefficients $A, B$, and $C$ are given by

$$
\begin{aligned}
& A= m / \dot{\varepsilon}_{g} \\
& B=\gamma-\left(m / \dot{\varepsilon}_{g}\right)\left(D_{\ell \ell} \alpha+D_{t t}\right) \beta \\
& \quad-\alpha \beta D_{l}^{2}-\beta D_{t}^{2} \\
& C=-\gamma \dot{\varepsilon}_{g}\left(D_{\ell \ell} \alpha+D_{t t}\right) \beta \\
&+\alpha \beta^{2} \dot{\varepsilon}_{g}\left(D_{l}^{2} D_{t t}-2 D_{\ell} D_{t} D_{\ell t}+D_{t}^{2} D_{\ell \ell}\right)
\end{aligned}
$$

It may be noted that, when the second derivatives $D_{1 j}$ are assumed to be zero, Equation (20) becomes

$$
\frac{m}{\dot{\varepsilon}_{g}} \frac{d^{2}}{d t^{2}}\left(\delta \varepsilon_{g}\right)+\left(\gamma-\alpha \beta D_{l}^{2}\right) \frac{d}{d t}\left(\delta \varepsilon_{g}\right)=0
$$

This equation gives the same condition for $a b-$ solute stability (zero roots) as Hillier 
(Reference 4) for his special case (ii) with $\mathrm{R}=1, \mathrm{P}=1, \mathrm{~m}=0$, i.e.,

$$
\left(\gamma_{0}-\alpha \beta D_{l}^{2}-\beta D_{t}^{2}\right)=0
$$

which specifies the critical value of $\gamma$ for specified values of stress conditions defined by $\alpha$ and $\beta$. The subscript has been added to $\gamma$ to indicate that $\gamma_{0}$ is the special value of $\gamma$ which leads to absolute stability if second derivatives of $\sigma_{g}$, i.e., $D_{i j}$, are assumed to be zero. It may be noted that the roots of Equation (24) are linearly dependent on $\dot{\epsilon}_{\mathrm{g}} / \mathrm{m}$. The solution to Equation (24) is of the form

$$
\delta \dot{\varepsilon}_{g}=\left(\delta \dot{\varepsilon}_{g}\right)_{0} \exp \left[\left(\gamma-\alpha \beta D_{l}^{2}-\beta D_{t}^{2}\right)\left(\dot{\varepsilon}_{g} / m\right) \Delta t\right]
$$

where $\left(\delta \dot{\epsilon}_{\mathrm{g}}\right)_{0}$ is the initial value of $\delta \dot{\epsilon}_{\mathrm{g}}$ at time $\Delta t=0$. The time constant for the growth or dimunition of the perturbation $\delta \dot{\epsilon}_{g}$ is, not unexpectedly, proportional to the strain rate sensitivity $m$ and inversely proportional to the nominal strain rate $\dot{\epsilon}_{\mathrm{g}}$.

Turning now to the full solution of Equation (20) including terms in $D_{1]}$ the two roots are

$$
\lambda=\frac{-B \pm \sqrt{B^{2}-4 . A C}}{2 A}
$$

With $\bar{A}=\mathrm{m} / \dot{\epsilon}_{\mathrm{g}}$ it is clear that the time constants of increase or decay in $\delta \dot{\epsilon}_{g}$ are again linearly related to $\epsilon_{g}$ and inversely to $m$. However, since the numerator in Equation (27) is also a function of $m$ (though not of $\dot{\epsilon}_{g}$ ) the m-dependence for $\lambda$ as a whole is not strictly linear, though this will be nearly so when $m$ is small enough.

The positive sign in Equation (27) is identified with the most positive root since both the factors $m$ and $\dot{\epsilon}_{\mathrm{g}}$ of $A$ in Equation (2l) are positive ( $m$ is positive for realistic properties). The condition for absolute stability becomes

$$
\sqrt{B^{2}-4 A C}<B
$$

or, with $\AA$ and $B$ positive,

$$
\mathrm{C}<\mathrm{O}
$$

If $B$ is negative, stability is unattainable.

\section{DISCUSSION OF RESULTS}

\section{A. Roots of the Characteristic Equation}

Values of the normalized root $\lambda / \dot{\epsilon}_{g}$ are shown in Figures 2 through 7 for a range of values of $\alpha=\sigma_{\ell} / \sigma_{\mathrm{t}}$ and strain hardening parameter $\cdot \gamma$ for typical values of $R, P$, and strain rate sensitivity $m$. Some of these figures show the smaller root, Equation (27), in addition to the larger root although the algebraically larger root determines stability. Observations are as follows:

1. Stability is always reduced ( $\lambda$ is more positive) as the value of $\gamma$ is decreased. This follows the direction of the classical stability criterion of Considere (Reference 10) for the uniaxial case without $m$-dependence,

$$
\gamma=\frac{1}{\sigma} \frac{d \sigma}{\partial \varepsilon}<1
$$

2. As the strain rate sensitivity $m$ increases, stability is increased in the sense that the roots $\lambda$ become reduced.

3. The above conclusions are true for anisotropic properties $(\mathrm{R}>1, \mathrm{P}>1)$. If $\mathrm{R} \neq \mathrm{P}$ the roots $\lambda$ are not the same when $\alpha$ is replaced by $1 / \alpha$.

4. In certain regions of $\alpha$, absolute stability is never attained (roots are always positive). At first sight this seems paradoxical, but this only implies that inhomogeneities will always propagate, though perhaps at negligible rates.

To determine conditions for propagation of inhomogeneities at acceptable rates, a solution to Equation (20) of the form

$$
\delta \dot{\varepsilon}_{g} \cong K_{I} \exp \left[\left(\lambda / \dot{\varepsilon}_{g}\right) \dot{\varepsilon}_{g} \Delta t\right]
$$

is considered, where $\lambda$ is the largest root in Equation (27). An acceptably small value of $\lambda / \dot{\epsilon}_{g}$ can be defined as that value which makes the exponential factor in Equations (31) acceptably small, say e $=2.718$ for convenience, after some acceptable time $\Delta t$. As can be seen from the form of Equation (31), if the normalized root $\lambda / \dot{\epsilon}_{\mathrm{g}}$ is taken, the remaining term in the exponent is $\dot{\epsilon}_{\mathrm{g}} \Delta \mathrm{t}$, or $\Delta \boldsymbol{\epsilon}_{\mathrm{g}}$ within the assumptions of constancy of $\dot{\epsilon}_{g}$. Now in practical cases it is sufficient to consider the growth of an inhomogeneity only for some specific maximum strain (here the thinning) of the material outside the inhomogeneity. If this maximum strain is taken as 10 percent then the allowable value of $\lambda / \dot{\epsilon}_{\mathrm{g}}$ is determined by

$$
2.718=\exp \left[\left(\lambda / \dot{\varepsilon}_{g}\right) / 10\right]
$$

or

$$
\left(\lambda / \dot{\varepsilon}_{\mathrm{g}}\right)_{\text {eff }}=10
$$

where $\left(\lambda / \dot{\boldsymbol{\epsilon}}_{\mathrm{g}}\right)$ eff is thus the value of $\lambda / \dot{\boldsymbol{\epsilon}}_{\mathrm{g}}$ which makes the perturbation strain rate increase by a 
factor of $\theta=2.718$ when a nominal strain increment of 10 percent is reached in the surrounding material. These values of $\lambda / \dot{\epsilon}_{g}$ are labeled as the effective stability points in Figures 2 through 7. It can be seen that effective stability is achieved, i.e., no "substantial" aggravation of a neck, for values of $\gamma=\gamma$ eff often considerably lower than those for absolute stability.

\section{B. Critical Values of $\gamma$ and $m$}

Values of the reciprocal strain hardening parameter $\gamma^{-1}=\sigma_{q} /\left(\partial \sigma_{g} / \partial \epsilon_{q}\right)$ for absolute stability $\left(\gamma-\frac{1}{a b s}\right)$ and for effective stability $\left(\gamma_{-1}\right)$ are plotted in Figures 8, 9, and 10. These three figures relate to isotropic properties $(R=P=1)$, to anisotropic properties $(R=2, P=4)$ and to anistropic properties with isotropy between the width and thickness directions $(R=4, P=4)$. Variations with strain rate sensitivity $m$ are also shown in these figures. The value $\gamma_{0}^{-1}$ for stability, assuming second derivatives $D_{i j}$ are zero, from Equations (24) and (25) is also shown. The same comments are applicable as those made in connection with the plots of $\lambda$ in Figures 2 to 7 .

In addition it may be seen that the values of $\gamma$ of are close to those of $\gamma_{0}^{-1}$ for small values of $m$, say $m \leqslant 0.01$. This is not a coincidence as will now be demonstrated.

If the roots $\lambda$ of Equation (20) are examined for $m \longrightarrow 0$, in the range $C<0$ (see Figures 8, 9 , and 10), assuming as betore that $A$ from Equation (21) is positive, Equation (27) shows that the largest (most positive) values of $\lambda$ approach $|C| / B$ if $B$ is positive. As B goes through zero, provided $A$ is finite, $\lambda$ becomes $(|C| / A)^{1 / 2}$. However, when B becomes negative, with $m$ still vanishingly small, the largest root is now $|B| / A$. Thus, a change in sign of $B$ causes the root to go from a low positive value to a high positive value, and therefore the effective stability point would be expected to be in the region close to $B=0$ for small values of $m$.

\section{vI. CONCLUSIONS}

1. Inclusions of first order changes in the strain director inside the patch causes the characteristic equation governing stability to become quadratic whereas exclusion of these changes produces a first order equation.

2. The roots of the quadratic equation are real in all practical cases.

3. For some values of biaxiality ratio $\sigma_{l} / \sigma_{1}$ the largest root is positive for all values of strain rate sensitivity $m$ and strain hardening parameter $\gamma$.

4. Stability can be redefined as a condition whereby "significant" neck growth does not occur for some prescribed strain (e.g. 10 percent) in the material outside the patch. The effective value of strain hardening parameter, $\gamma$ eff, for this condition and for $m \rightarrow 0$ is very close to the value obtained without inclusion of changes in strain directors. This is shown to be a rational conclusion.

5. As the strain rate sensitivity $m$ is increased beyond about 0.1 the values of $\gamma_{\text {ett }}$ become very small, indicating high relative stability.

6. Effects of anisotropy are not large over the normal ranges.

\section{REFERENCES}

1. E. Duncombe, "Plastic Instability and Growth of Grooves and Patches in Plates or Tubes," Int. J. Mech. Sci., 14, pp 325-337, 1972.

2. W. T. Lankford and E. Saibel, "Some Problems in Unstable Plastic Flow Under Biaxial Tension," American Institute of Mining and Metallurgical Engineers, Technical Publication No. 2238, Class E, Metals Technology, August 1947.

3. H. W. Swift, "Plastic Instability Under Plane Stress," Journal of the Mechanics and Physics of Solids, 1, pp 1 to 18, 1952.

4. M. J. Hillier, "Tensile Instability of Thin Tubes - I," Int. J. Mech. Sci., 7, pp 531-538, 1965.

5. R. Hill, "The Mathematical Theory of Plasticity," pp 317-320, Clarendon Press - Oxford (1950).

6. D. C. Bogue, "The Yield Stress and Plastic Strain Theory for Anisotropic Materials," Oak Ridge National Laboratory Report ORNL-TM-1869, July 1967.

7. A. M. Lyapunov, "Problème Général de la Stabilité de Mouvement," Annals of Mathematical Studies Number 17, Princeton University Press, 1949.

8. S. P. Keeler and W. A. Backofen, "Plastic Instability and Fracture in Sheets Stretched Over Rigid Punches," Transactions of the $A S M, 56$, pp 25-48, 1963.

9. M. Azrin and W. A. Backofen, "The Deformation and Failure of a Biaxially Stretched Sheet," Metallurgical Transactions, 1, pp 2857-2865, 1970.

10. M. Considère, "Memoire sur l'Emploi du Fer et l'Acier dans les Constructions," Ann. des Ponts et Chaussees, 9, Ser. 6, p. 574, 1885. 

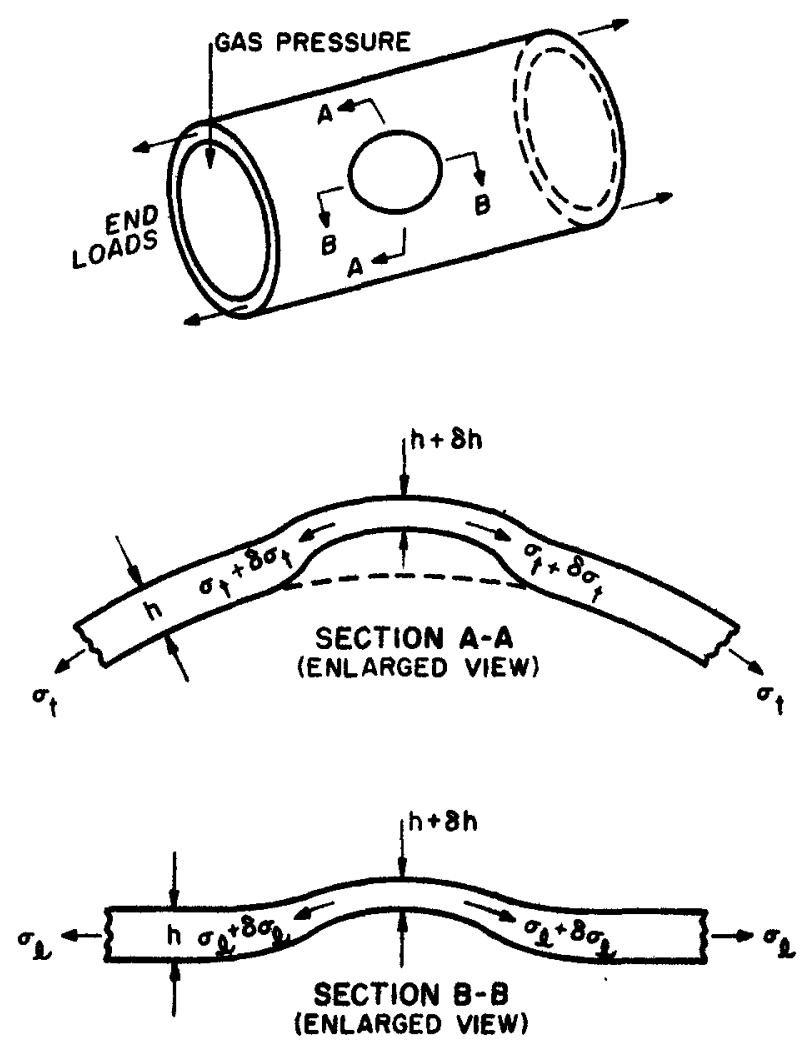

Figure 1. Locally Thinned Region of a Tube Subjected to Internal Gas Pressure and End Loads

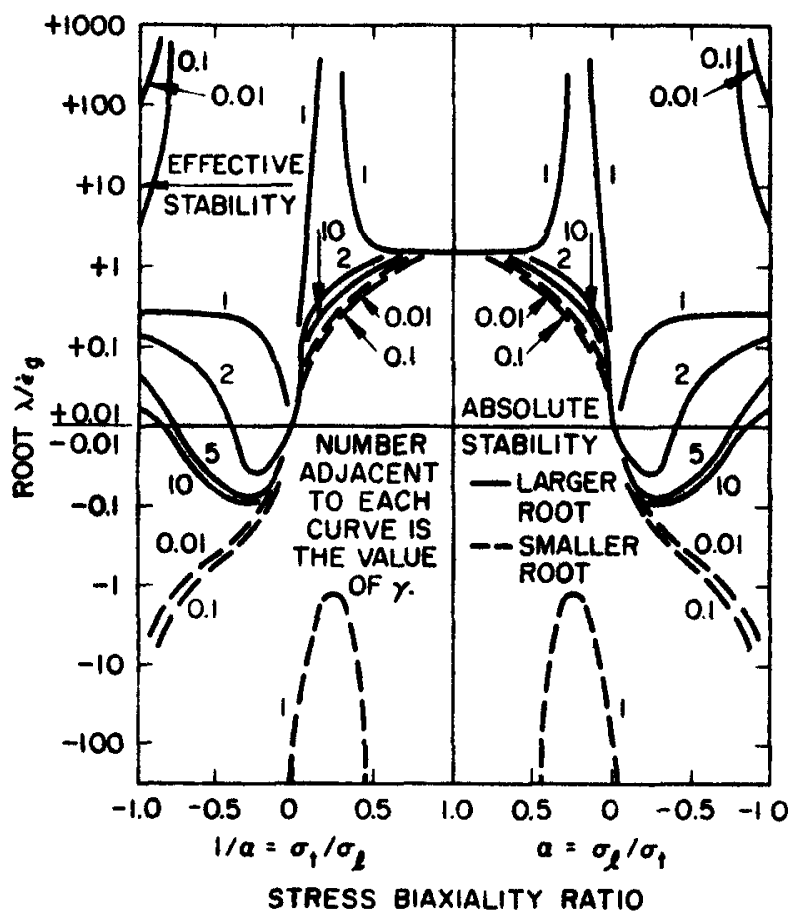

Figure 2. Roots of Equation for Neck Growth, $R=P=1, \mathrm{~m}=10^{-5}$

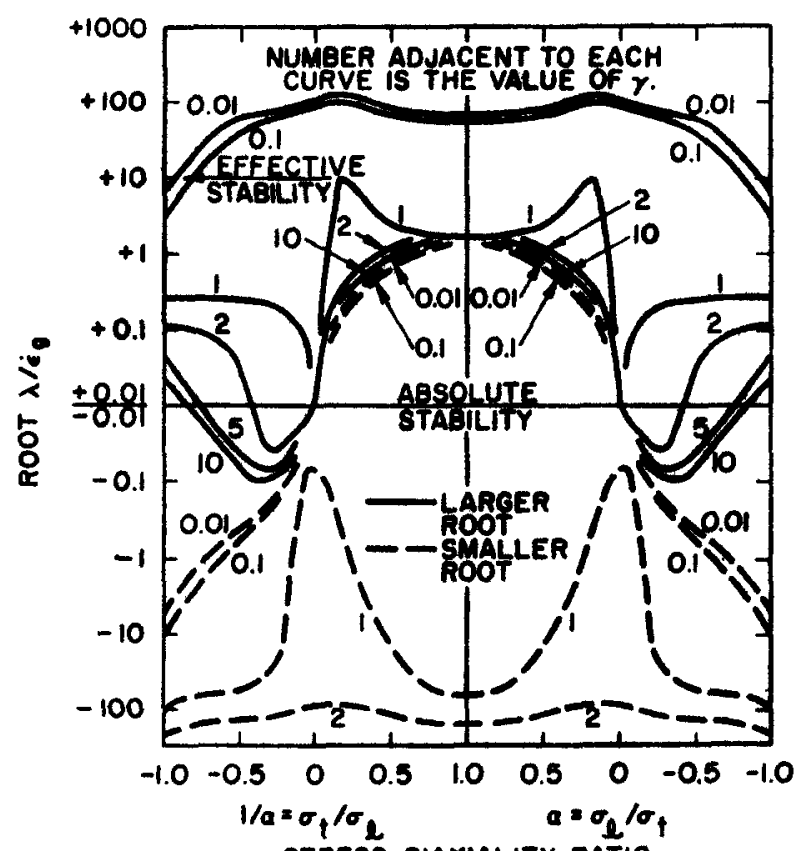

STRESS BIAXIALITY RATIO

Figure 3. Roots of Equation for Nook Growth, $R=P=1, \mathrm{~m}=0.01$ 


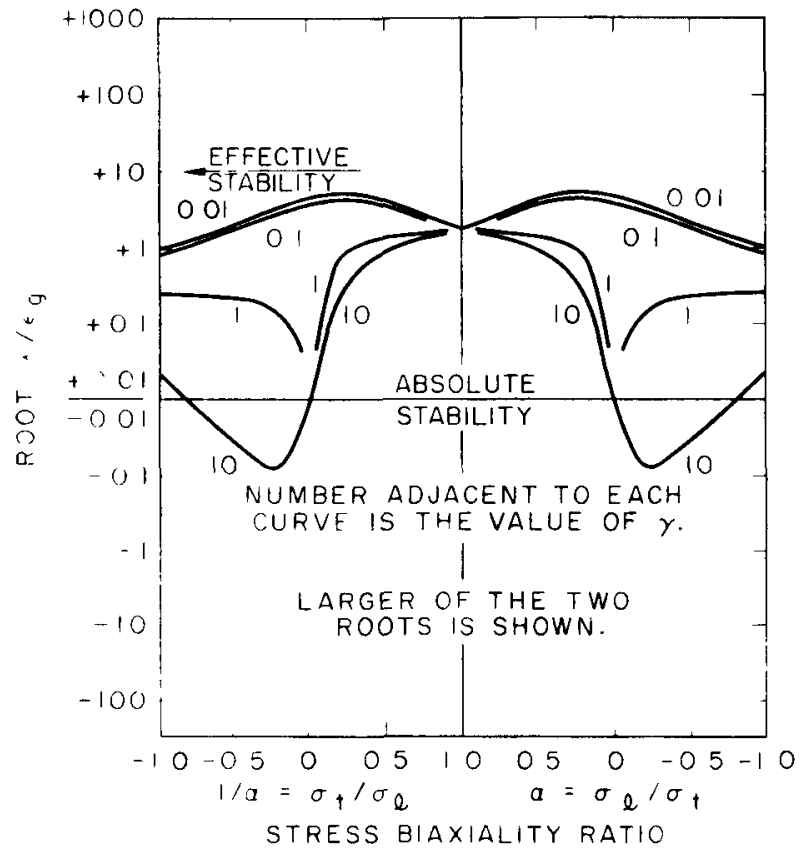

Figure 4. Roots of Equation for Neck Growth, $R=P=1, m=0.25$

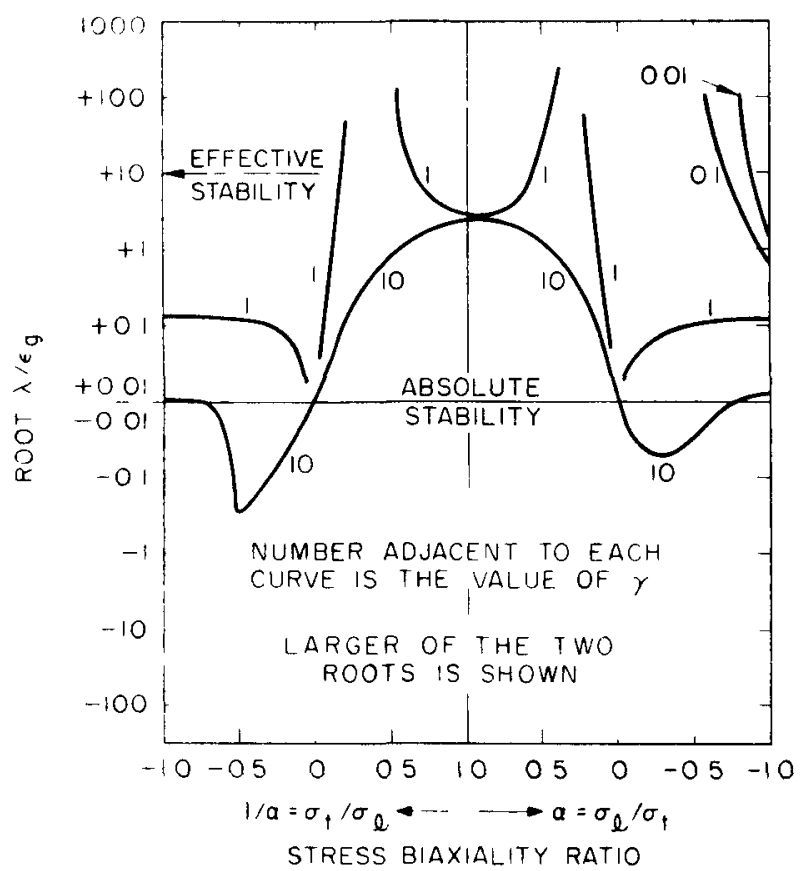

Figure 6. Roots of Equation for Neck Growth, $R=2, P=4, m=10^{-5}$

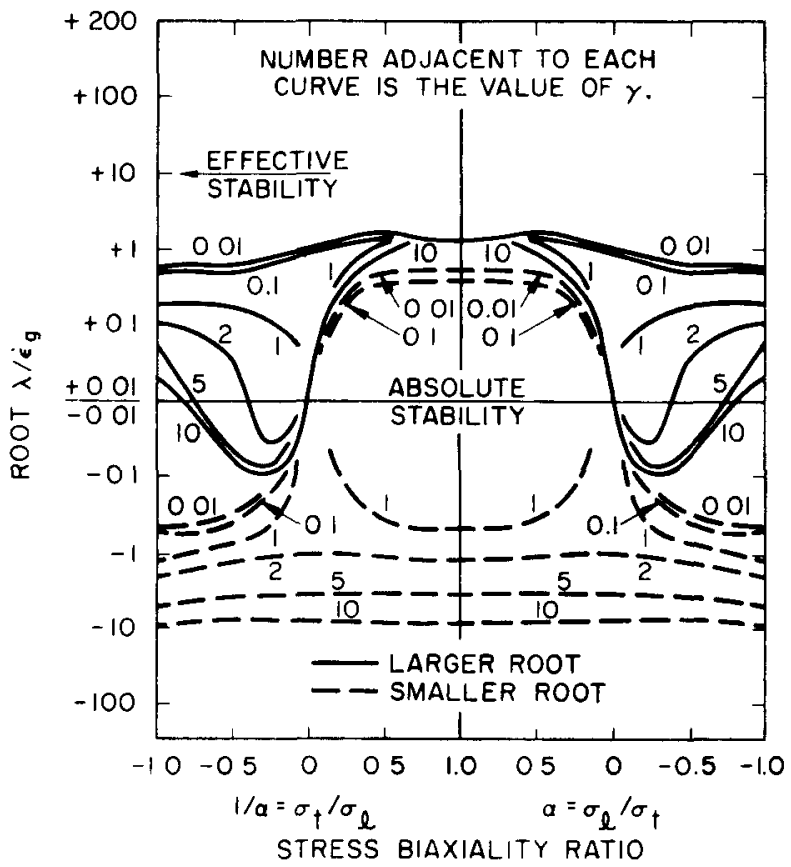

Figure 5. Roots of Equation for Neck Growth, $\mathbf{R}=\mathbf{P}=1, \mathbf{m}=1$

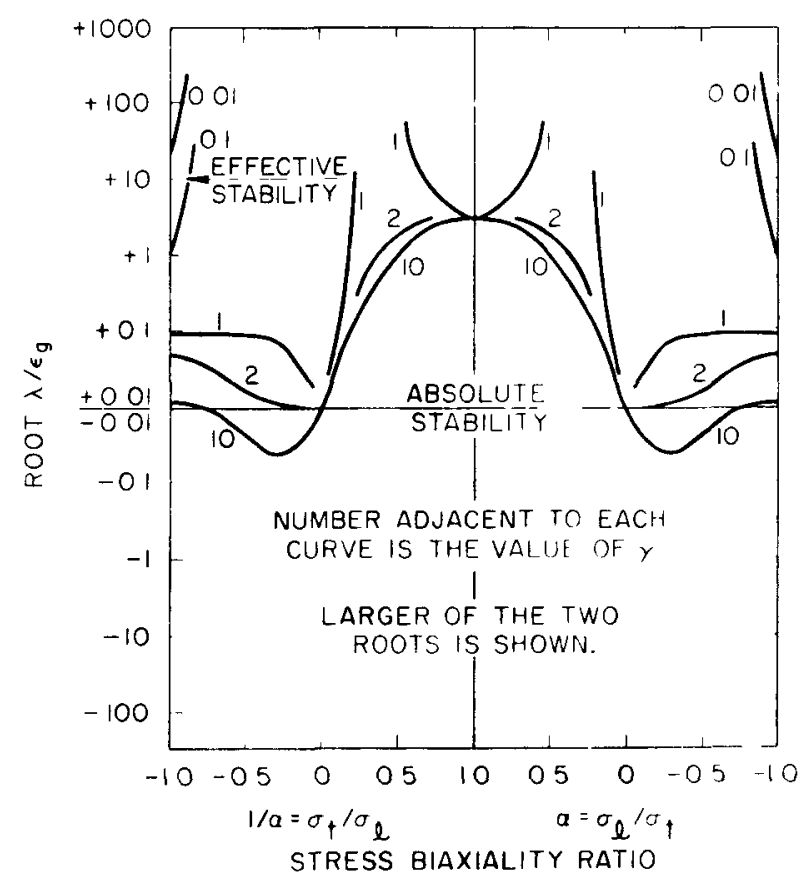

Figure 7. Roots of Equation for Neck Growth, $R=4, P=4, m=10^{-5}$ 


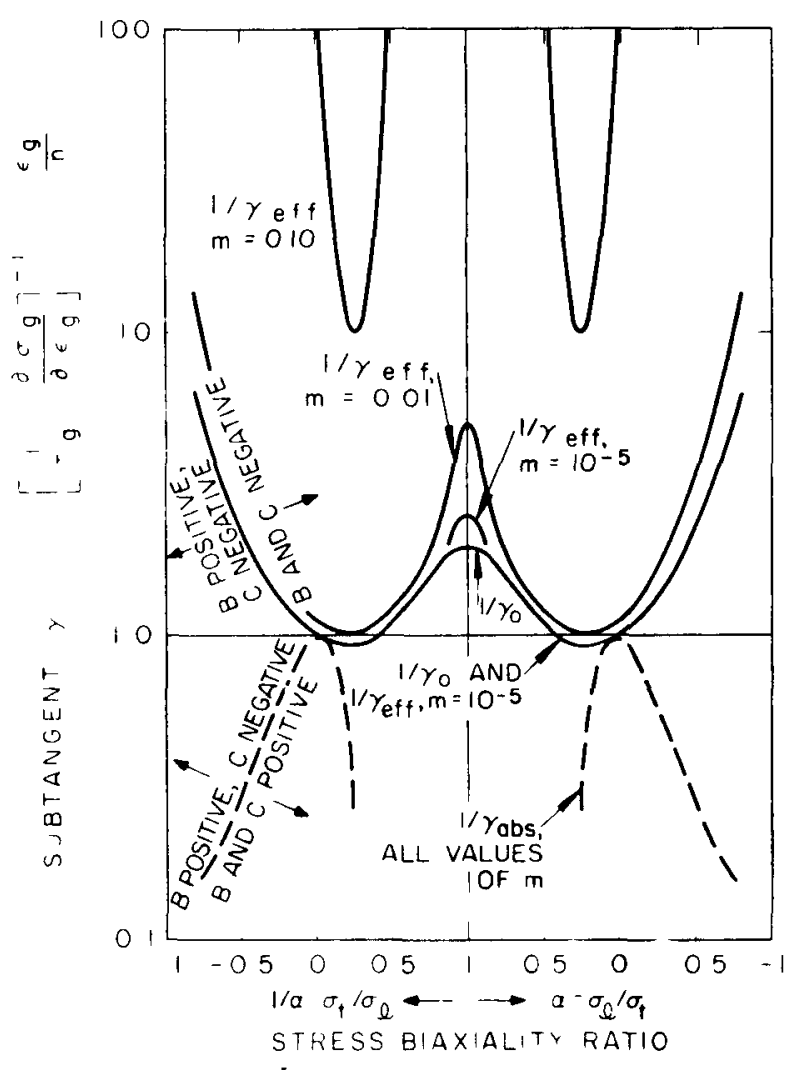

Figure 8. Absolute and Effective Stability Criteria, $\mathbf{R}=\mathbf{P}=\mathbf{I}$

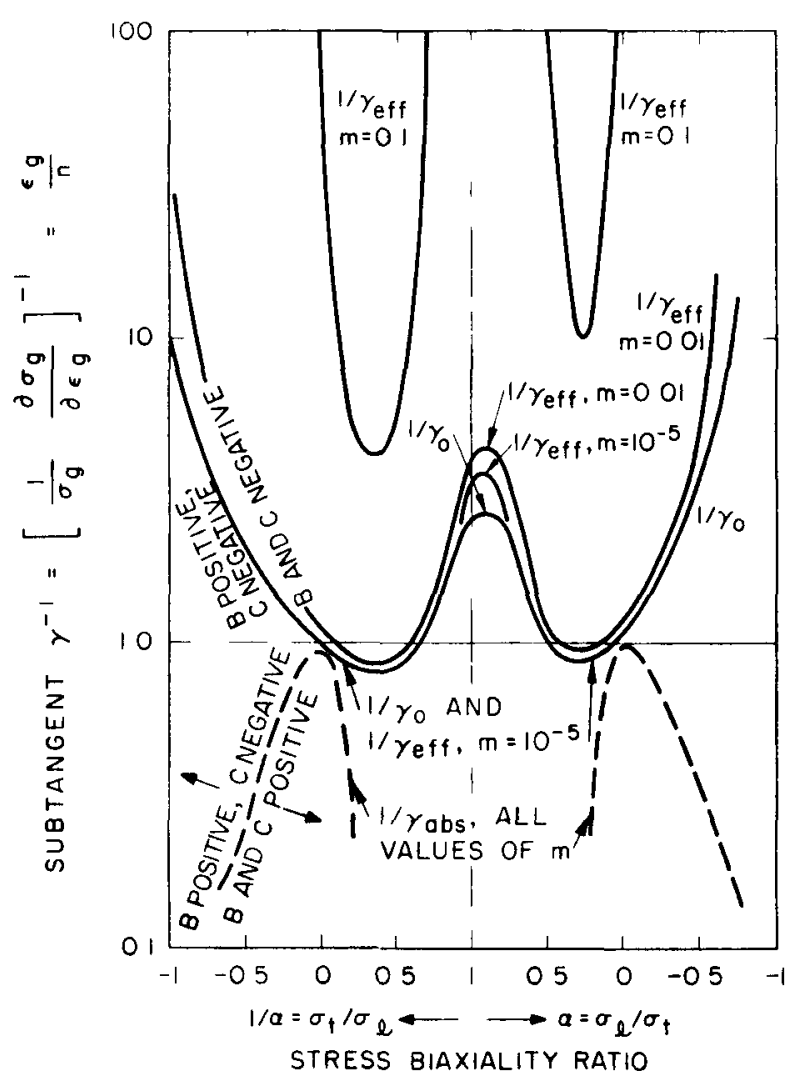

Figure 9. Absolute and Effective Stability Criteria, $R=2, P=4$

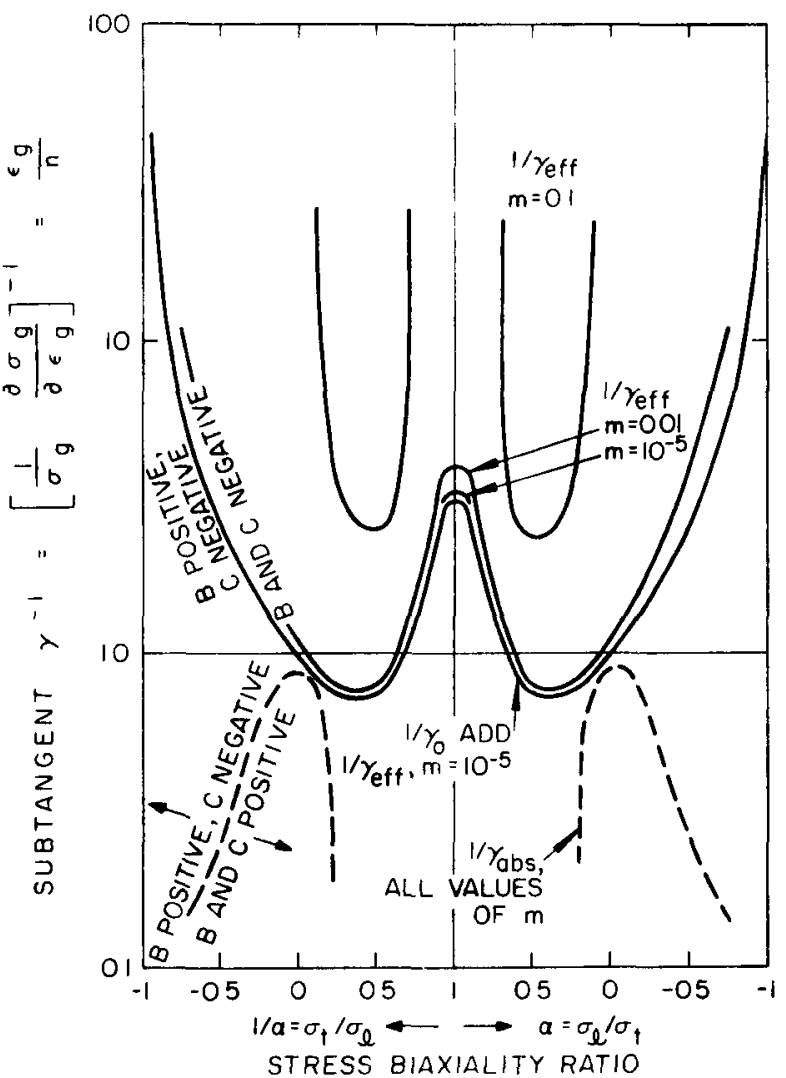

Figure 10. Absolute and Effective Stability Criteria, $R=P=4$ 


\section{APPENDIX A}

\section{DERIVATION OF THE CHARACTERISTIC EQUATION FOR STABILITY}

The perturbation variables can be identified as $\delta \sigma_{\mathrm{g}}, \delta \sigma_{\ell}, \delta \sigma_{\mathrm{t}}, \delta \epsilon_{\ell}, \delta \epsilon_{\mathrm{t}}, \delta \epsilon_{\mathrm{g}}$. The time derivatives of variables, such as $\delta \dot{\epsilon}_{g}$, are not treated here as new variables, but as $\boldsymbol{S} \delta \dot{\boldsymbol{\epsilon}}_{\mathrm{g}}$ where the multiplier $S$ can be considered as a linear operator, or equivalently, as Laplace transform multiplier. For solution of this system there is first the constitutive Equation (1). In addition, there are two boundary conditions, Equations (18) and (19). Then there is the relation, Equation (2), between $\sigma_{g}, \sigma_{\ell}$, and $\sigma_{\mathrm{t}}$. This can be expressed in perturbation variables as

$$
\begin{aligned}
\delta \sigma_{g} & =\frac{\partial \sigma_{g}}{\partial \sigma_{\ell}} \delta \sigma_{\ell}+\frac{\partial \sigma_{g}}{\partial \sigma_{t}} \delta \sigma_{t} \\
& =D_{\ell} \delta \sigma_{\ell}+D_{t} \delta \sigma_{t}
\end{aligned}
$$

Finally, the two relations, Equations (5) and (6), exist between $\dot{\boldsymbol{\epsilon}}_{\ell}$ and $\dot{\boldsymbol{\epsilon}}_{\mathrm{g}}$ and between $\dot{\boldsymbol{\epsilon}}_{\mathrm{t}}$ and $\dot{\epsilon}_{g}$. These can be expressed in perturbation form as

$$
\begin{aligned}
\delta \varepsilon_{\ell} & =\delta\left(D_{\ell} \dot{\varepsilon}_{g}\right)=D_{\ell} \delta \dot{\varepsilon}_{B}+\dot{\varepsilon}_{F} \delta D_{\ell} \\
& =I_{\ell} \delta \dot{\varepsilon}_{E}+\dot{\varepsilon}_{g}\left(\frac{\partial D_{\ell}}{\delta \sigma_{\ell}} \delta \sigma_{g}+\frac{\partial D_{\ell}}{\partial \sigma_{t}} \delta r_{t}\right) \\
& =D_{\ell} \delta \dot{\varepsilon}_{g}+\dot{\varepsilon}_{g}\left(D_{\ell \ell} \frac{\sigma_{\ell}}{\sigma_{g}} \frac{\delta \sigma_{\ell}}{\sigma_{l}}+D_{\ell t} \frac{\sigma_{t}}{\sigma_{g}} \frac{\delta \sigma_{t}}{\sigma_{t}}\right)
\end{aligned}
$$

where $\mathrm{D}_{\ell \ell}=\sigma_{g} \partial \mathrm{D}_{\ell} / \partial \sigma_{\ell}$

and $D_{\ell t}=\sigma_{g} \partial D_{\ell} / \partial \sigma_{l}$, as given by Equations (13) and (15), and similarly

$$
\delta \dot{\varepsilon}_{t}=D_{t} \delta \dot{\varepsilon}_{g}+\dot{\varepsilon}_{B}\left(D_{t \ell} \frac{\sigma_{l}}{\sigma_{\varepsilon}} \frac{\delta \sigma_{l}}{\sigma_{l}}+D_{t}+\frac{\sigma_{t}}{\sigma_{g}} \frac{\delta \sigma_{t}}{\sigma_{t}}\right)
$$

where $D_{\mathrm{tt}}=\sigma_{\mathrm{g}} \quad \partial \mathrm{D}_{\mathrm{t}} / \partial \sigma_{\ell}=\mathrm{D}_{\mathrm{lt}} \quad$ and $\quad \mathrm{D}_{\mathrm{tt}}=$ $\sigma_{\mathrm{g}} \partial \mathrm{D}_{\mathrm{t}} / \partial \sigma_{\mathrm{t}}$ as given by Equations (14) and (15).

Equations (1), (18), (19), ( $\mathbb{A}-1),(\mathbb{A}-2)$, and $(A-3)$ are thus sufficient to find a solution. The solution equation will be derived in $\delta \epsilon_{g}$.

The variable $\delta \sigma_{g}$ is first eliminated by combining Equation (1) and Equation ( $\mathbf{A}-1)$ into

$$
\frac{I}{\sigma_{g}}\left[D_{\ell} \delta \sigma_{\ell}+D_{t} \delta \sigma_{t}\right]=m \frac{\delta \dot{\varepsilon}_{g}}{\dot{\varepsilon}_{g}}+\gamma \delta \varepsilon_{g}
$$

where the new variable $\gamma$ is used instead of $n / \epsilon_{\mathrm{g}}$. The system is now reduced to Equations (18), (19), (A-2), (A-3), and (A-4) and to five variables $\delta \sigma_{\ell}, \delta \sigma_{\mathrm{t}}, \delta \epsilon_{\ell}, \delta \epsilon_{\mathrm{t}}$, and $\delta \epsilon_{\mathrm{g}}$. The next step is to use

Equations (18) and (19) to replace $\delta \sigma_{\ell}$ and $\delta \sigma_{t}$ by $\delta \epsilon_{\ell}$ and $\delta \epsilon_{\mathrm{t}}$. If this is performed in Equation (A-2) and (A-3), these equations become

$$
\begin{aligned}
& \delta \dot{\varepsilon}_{\ell}=D_{\ell} \delta \dot{\varepsilon}_{g}+\dot{\varepsilon}_{g}\left(D_{\ell \ell} \frac{\sigma_{l}}{\sigma_{g}} \delta \varepsilon_{\ell}+D_{\ell t} \frac{\sigma_{t}}{\sigma_{g}} \delta \varepsilon_{t}\right) \\
& \delta \dot{\varepsilon}_{t}=D_{t} \delta \dot{\varepsilon}_{g}+\dot{\varepsilon}_{g}\left(D_{t \ell} \frac{\sigma_{\ell}}{\sigma_{g}} \delta \varepsilon_{l}+D_{t t} \frac{\sigma_{t}}{\sigma_{g}} \delta \varepsilon_{t}\right)
\end{aligned}
$$

This reduces the variables to $\delta \epsilon_{\ell}, \delta \epsilon_{t}$, and $\delta \epsilon_{q}$ and the equations to $(\AA-4),(A-5)$ and $(\AA-6)$. It is now necessary to solve Equations ( $A-5)$ and (A-6) simultaneously for $\delta \epsilon_{\mathfrak{l}}$ and $\delta \epsilon_{\mathrm{t}}$ as functions of $\delta \epsilon_{\mathfrak{g}}$ and then put each of these solutions into Equation (A-4). The simultaneous solution is

$$
\begin{aligned}
& \delta \varepsilon_{\ell}=\frac{\left(D_{\ell} \delta \dot{\varepsilon}_{g}\right)\left(S-\varepsilon_{g} \alpha \beta D_{t t}\right)+\left(\dot{\varepsilon}_{g} D_{t t} a \beta\right)\left(D_{t} \delta \dot{\varepsilon}_{g}\right)}{\left(S-\varepsilon_{g} D_{\ell \ell} a\right)\left(\varepsilon-\varepsilon_{g} D_{t t} a B\right)-\dot{\varepsilon}_{g}^{2} D_{l t}^{2} \alpha^{2} \beta}
\end{aligned}
$$

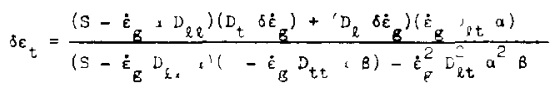

where $\alpha=\sigma / \sigma_{\mathfrak{t}}, \beta=\sigma_{\mathfrak{t}} / \sigma_{\mathrm{g}}$, and $S$ is the time operator $\mathrm{d} / \mathrm{dt}$.

Finally, the above expressions for $\epsilon_{\ell}$ and $\epsilon_{t}$ can be inserted into Equation ( $A-4)$, yielding a differential equation in $\epsilon_{g}$ only. Some simplification occurs in the denominators of Equations (A-7) and (A-8) owing to the identity

$$
D_{t t} D_{l \ell}=D_{t \ell}^{2}
$$

which can be shown by using Equations (13), (14), and (15). The final equation of motion for $\delta \dot{\boldsymbol{\epsilon}}_{\mathrm{g}}$ is second order and of the form

$$
\left(A S^{2}+B S+C\right) \delta \dot{\varepsilon}_{g}=0
$$

where

$$
\begin{aligned}
& \mathrm{A}=\mathrm{m} / \dot{\varepsilon}_{\mathrm{g}} \\
& B=\gamma-\left(m / \dot{\varepsilon}_{g}\right)\left(D_{\ell \ell} \alpha+D_{t t}\right) B \\
& -\alpha \beta D_{l}^{2}-\beta D_{t}^{2} \\
& C=-\gamma \dot{\varepsilon}_{g}\left(D_{\ell \ell} \alpha+D_{t t}\right) B \\
& +\alpha \beta^{2} \dot{\varepsilon}_{g}\left(D_{\ell}^{2} D_{t t}-2 D_{\ell} D_{t} D_{l t}+D_{t}^{2} D_{\ell l}\right)
\end{aligned}
$$

